\title{
Kingfish Striations and the Kelvin-Helmholtz Instability: Part 1
}

James H. Hunter, Jr.*

\section{DISCLAIMER}

This report was prepared as an account of work sponsored by an agency of the United States Government. Neither the United States Government nor any agency thereof, nor any of thieir employees, makes any warranty, express or implied, or assumes any legal liability or responsibility for the accuracy, completeness, or usefulness of any information, apparatus, product, or process disclosed, or represents that its use svould not infringe privately owned rights. Reference herein to any specific commercial product, process, or service by trade name, trademark, manufacturer, or otherwise does not necessarily constitute or imply its endorsement, recommendation, or favoring by the United States Government or any agency thereof. The views and opinions of authors expressed herein do not necessarily state or reflect those of the United States Governinent or any agency thereof.

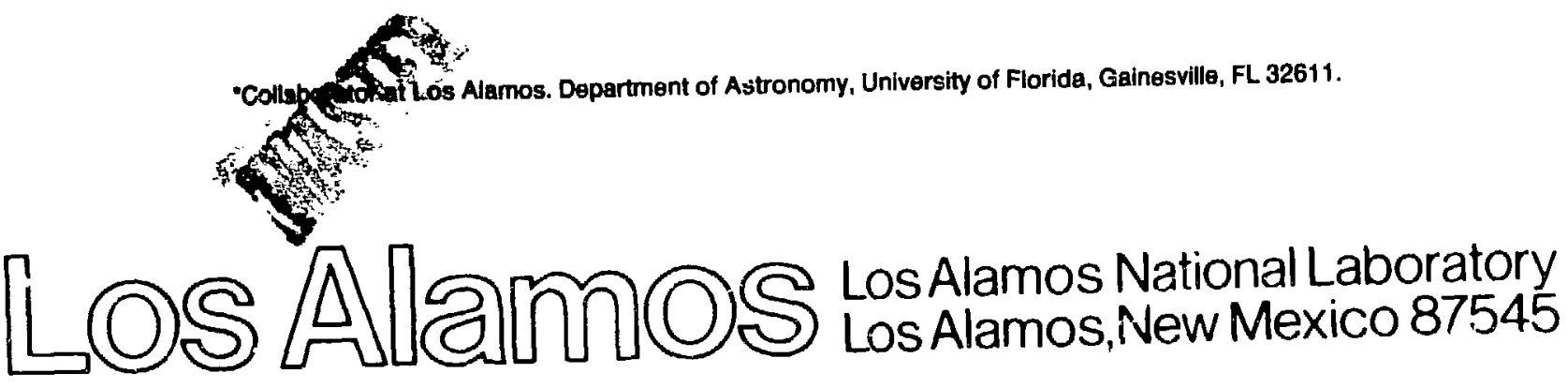


KINGFISH STRIATIONS AND THE KELVIN-HELMHOLTZ INSTABILITY: PART 1

by

James $H$. Hun ter, Jr.

\section{ABSTRACT}

The role of the Kelvin-Helmholtz instability in initiating the formation of the density striations observed in the Kingfish fireball is examined. Two idealized models are proposed for the velocity shear layer on the sides of the fireball, each of which includes essential characieristics of the Kingfish event insofar as the development of Kelvin-He lmholtz instabllities is concerned. A complete linear analysis is presented for each model.

\section{INTRODUCTION}

The photographic records of the Kingfish fireball reveal the presence of regularly spaced striations aligned along the magnetic fields on the surface of the firebail. The strlations appear first, and grow most rapidiy, on the sides of the Eireball. They appear dramatically at a well-established time af ter the detonation. The approximate e-folding time for the instability is knowt.

Detalled numerical simulations of the rising kingfish firebell show that, when the siriations appear, positive ions (princlpaliy $0^{+}$) outside the fireball, but behind the shock, would be rigidly attached to the magnetic field and therefore would slip past the regions on the fireball where the striations are observed. Essentlally, the slip speed would be that of the rising fireba11, U. Moreover, estimates show that the resulting shear layer yould be thin, having a thickness considerably less than the separation between adjacent striations. The velocity shear ard slip velocity would be greatest on the sides of the firebell where the striations first appear. This suggests that the striations may be caused (i.e., initiated) by a Kelvin-Helmholtz instability. On morphoiogical grounds, a Rayleigh-Taylor instability seems 
less likely because that inscabllity would be expected to grow most rapidly on the top of the Eireball where the fluld deceleration is greatest.

Our purpose in this report is to review selected aspects of linear KelvinHelmholtz instability theory in the context of the kingfish event. In Section II, we formulate an idealized problem, which retains the essential ingredients of the Kingfish event insofar as the development of KelvinHe lmholtz instabllities is concerned. In Sections III and IV, we consider carefully two models, each of which can be analyzed in a straightforward fashion without resorting to extensive numerical calculations. Although idealized, each of these models has a direct beaing on the problem at hand.

II. FORMULATION OF THE PROBLEM

In this section, we cunsider the Kelvin-Helmholtz instability (hereafter, designated $\mathrm{K}-\mathrm{H}$ Instability) in a compressible, magnetized fluld. The ideal MHD equations are

$$
\begin{aligned}
& \frac{\mathrm{d} \rho}{\mathrm{dt}}=-\underset{\sim}{\nabla} \cdot \rho \underset{\sim}{\mathrm{V}}, \\
& \rho \frac{d V}{d t}=-\underset{\sim}{D}\left(p+\frac{B^{2}}{8 \pi}\right)+\frac{1}{4 \pi}(\underset{\sim}{B} \cdot \underset{\sim}{\nabla} \underset{\sim}{B}+\underset{\sim}{ \pm}, \\
& \frac{\partial \underline{B}}{\partial t}=\underline{\nabla} \times(\underset{\sim}{V} \times \underset{\sim}{B})
\end{aligned}
$$

and

$$
\frac{d p}{d t}=\frac{Y_{g} p}{p} \frac{d p}{d t},
$$

where $p$ is the density, $\underset{\sim}{V}$ the bulk velocity, $\underset{\sim}{B}$ the magnetic field strength, $P$ the fluld pressure, and $\gamma_{g}$ the adlabatic index. The $f$ vector represents additional body forcas per unit mass, and the convective derivative $\mathrm{d} / \mathrm{dt}=\partial / \partial \mathrm{t}+\underline{\mathrm{V}} \cdot \underline{\nabla}$. Because the observed structures are relatively small 
compared with the fireball radius, the linear problem will be formulated in Cartesian coordinates. In the sollowing development, we adopt the conventional notation of Chandrasekhaz. 1 The physical situation is illustrated in Fig. 1 . In our problem, the undisturbed magnetic fleld, bulk velocity, and force

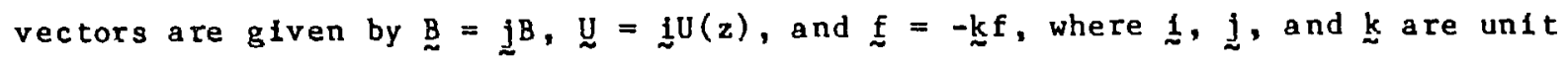
vectors in the $x, y$, and $z$ directions respectively. The equilibrium condition requires that $D\left(p+B^{2} / 8 \pi\right)=f$, where $D \equiv d / d z$. In formulating the ldeslized problem, $f$ will be zero, meaning that gradients are allowed only in $U(z)$. Therefore, in a formal sense, unperturbed pressure eq"ilibrlum will be imposed; $p+B^{2} / 8 \pi=$ constant. In reality, gradients exist in $P, B$, and $\rho$, due to the acceleration of the Earth's gravity, and to dynamical forces associated with the rising fireball. Therefore, to apply linear analysis to the Kingfish problem, we will use model results to gain insight into the behaviors of compressible $\mathrm{K}-\mathrm{H}$ instabilities. In Section III we will examine idealized models in which the undisturbed media are in pressure equilibrium.

The linearization of Equations (1)-(4) is straightforward and has been outlined in sone detail by Miura and Pritchett ${ }^{2}$ (hereafter, designated by MP). They Fourier analyze the linear, dependent variables. In our notation, this analysis requires that any perturbed quantity $\xi^{-}=\xi^{-}(z) \exp \left[1\left(k_{1} x+k_{2} y+n t\right)\right]$. When $f=0$, thelr second-order eigenvalue equation for the total pressure perturbation is $\mathrm{p}^{-*}=\mathrm{p}^{-}+\underset{\sim}{\mathrm{B}} \cdot \underline{\sim}^{-} / 4_{4} \pi$, which reduces io

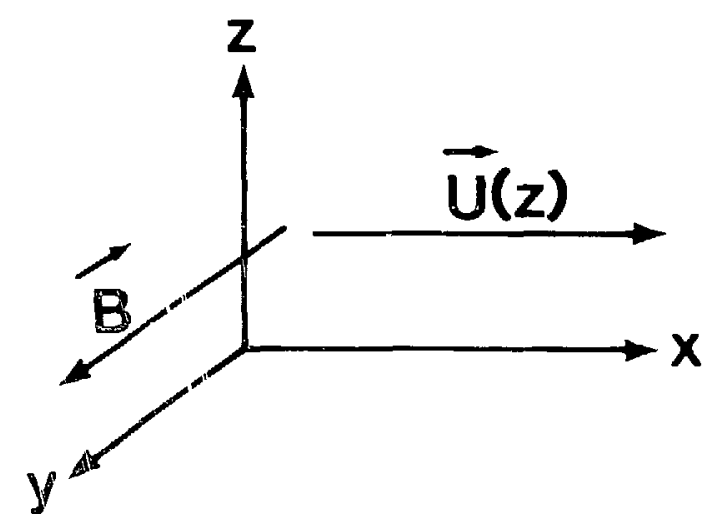

Fig. 1. The coordinate system used in the calculations. Inhomogeneities occur in the $z$ direction on ly. 


$$
\phi^{2} g D\left(\frac{1}{\phi^{2} g} D p^{-} \star\right)-\left[k_{1}^{2}+k_{2}^{2}-\frac{\phi^{2}}{\left(g c^{2}+v_{A}^{2}\right)}\right] p^{-} *=0,
$$

where $\phi=\left(n+k_{1} U\right)$ and $g=1-k_{3}^{2} v_{A}^{2} / \phi^{2}$. In Equation (5), the Alfven speed, $V_{A}$, and the adlabatic sound speed, $c$, in the undisturbed medium are deflaed by $v_{A}^{2}=B^{2} /(4 \pi \rho)$ and $c^{2}=\gamma_{g} p / \rho$. In our problem, the Alfven speed in the relatively tenuous, alipping medium is much greater than either or $U$. Consequently, the Mach number, $m=U / \sqrt{V_{A}^{2}+c^{2}}$, is small, and the gas will behave very nearly like an incompressible fluid. In the incompressible limtt, $K-H$ Instabilities are suppressed ${ }^{2}$ if $U<2 v_{A}(\underset{\sim}{k} \cdot \underline{B}) /\left(k_{1} B\right)$. In the present problem, in which $U / V_{A} \ll 1, K-H$ instabllities can develop oniy if $k$ is very nearly or thogonal to $\underset{\sim}{B}$. Therefore, in the following development, we restrict our attention to the most favorable (transverse) class of models in which the wave vectors are iliected parallel to the undisturbed flows; $\underset{\sim}{\mathbf{k}}=\underset{\sim}{\mathrm{i}} \mathrm{l}$. (We recall that the Kingfish striations, which correspond to density maxima, are aligned parallel to $B$, an observation which would follow if the transverse case applies.)

In the transverse case the linear equations describing our problem are

$$
\begin{aligned}
& \phi P^{-}=\frac{B}{4 \pi} \phi B_{y}^{-}-k_{1} c^{2} \rho u^{\prime}+i c^{2} \rho D_{w^{\prime}}, \\
& \rho \phi u^{\prime}=-k_{1} p^{-*}+1 \rho(D U)_{W^{-}}, \\
& \rho \phi w^{-}=1 D p^{-*} \text {, }
\end{aligned}
$$

and

$$
\phi B_{y}^{-j}=-k_{1} B u^{-}+1 B D w^{-}
$$

In these equations, $u^{\prime}$ and $w^{\prime}$ are the velocity perturbations in the $x$ and $z$ directions respectively, and $p^{-}=p^{-}+(B / 4 \pi) B_{y}^{-}=c^{2} p^{-}+(B / 4 \pi) B_{y}^{-}$. Equations (6) and (9) may be combined to give the result 


$$
\phi p^{-} \star=-k_{1} v^{2} \rho u^{-}+1 v^{2} \rho D w^{-}
$$

where $v^{2}=v_{A}^{2}+c^{2}$. Equations (7), (8), and (10) describe forms exactly the same as their hydrodynamic counterparts if the sound speed is replaced by $V$. Thus, the transverse hydromagnetic problem is formally equivalent to a hydrodynamic problem with $\underset{\sim}{\mathrm{k}}=\underset{\sim}{\mathbf{i}} \mathrm{k}_{1}$. However, the magnetized medium is less compressinle due to the presence of magnetic pressure.

We eliminate variables in the above system to obtain eigenvalue equations for both $p^{-}$a and $w^{-}$. The equation for $p^{-}$* reads

$$
\left\lceil D^{2}-\frac{2 k_{l}(D U) D}{\phi}+\frac{\phi^{2}}{v^{2}}-k_{1}^{2}\right\rceil p^{-}+=0,
$$

exactly the MP resilt when $k_{2}=k_{3}=0$. The elgenvalue equation for $w^{-}$is more difficult to derive; our result is

$$
\left\{D^{2}-\frac{2 \phi k_{1}(D U)}{\left(\phi^{2}-k_{1}^{2} v^{2}\right)} D+\left[\frac{\phi^{2}}{v^{2}}-k_{1}^{2}-\frac{k_{1}\left(D^{2} U\right)}{\phi}+\frac{2 k_{l}^{2}(D U)^{2}}{\left(\phi^{2}-k_{1}^{2} v^{2}\right)}\right]\right\} w^{-}=0
$$

To recover the incompressible $\lim 1 \mathrm{t}$, we let $V+\infty$. In that $1 \mathrm{im} i \mathrm{t}$, Equation (12) reduces to

$$
\left[\phi\left(D^{2}-k_{1}^{2}\right)-k_{1}\left(D^{2} U\right)\right] u^{-}=0,
$$

exactly Chandrasekhar's result for an incompressible fluid in the absence of unperturbed density gradients. It should be noted that Equations (11) and (12) have different forms. Generally speaking, the eigenvalue equations will be different for the various perturbed quantities when gradients are present in the undisturbed medium. If $U$ is constant, Equations (11) and (12) reduce to the identical forms: 


$$
\left(D^{2}-k_{1}^{2}+\frac{\phi^{2}}{v^{2}}\right)\left(p^{-*}, w^{-}\right)=0 \text {. }
$$

III. THE GENER

In a seminal paper, Gerwin ${ }^{3}$ considered the development of $K-H$ Instabilities at the interface between two compressible isotropic fluids having identical undisturbed densities and pressures, which flow relative to each other at a constant velocity parallel to the interface. In this section, we generalize Gervin's problem to include cases in which the densities in the media are unequal. The physical problem is 1 llustrated in Fig. 2. We adopt the convention that $\rho_{1}<\rho_{2}$. If the medin are in approximate pressure equilibrium, $\rho_{1} v_{1}^{2}=\rho_{2} v_{2}^{2}$.

In each medium, the total pressure perturbation must satisfy Equation (14). Since $U$ is elther constant or zero, the $\phi^{-} s$ are constant in both media. Consequently, the solutions of Equation (14) are of the form $\exp \pm(q z)$, where $q^{2}-k_{1}^{2}+\phi^{2} / v^{2}=0$. If $\left(n+k_{1} U\right)^{2} /\left(k_{1}^{2} v_{1}^{2}\right)$ and $n^{2} /\left(k_{1}^{2} v_{2}^{2}\right)<1$, then

$$
q_{1}=-k_{1}\left[1-\frac{\left(n+k_{1} u\right)^{2}}{k_{1}^{2} v_{1}^{2}}\right]^{1 / 2} \text {, }
$$

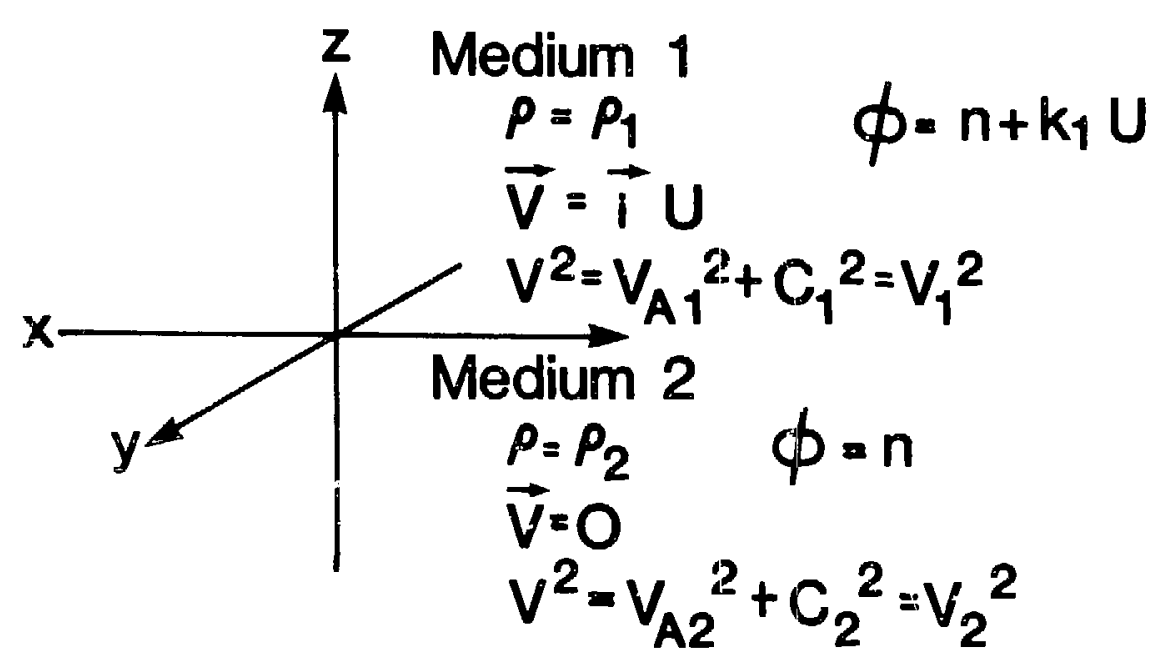

Fig. 2. The generalized Gerwin problem. 
and

$$
q_{2}=+k_{1}\left(1-\frac{n^{2}}{v_{2}^{2}}\right)^{1 / 2}
$$

The signs assoclated with $q_{1,2}$ follow from the requirement that the perturbations must vanish as $z+ \pm \infty$. [If $\phi_{1,2}^{2} /\left(k_{1}^{2} v_{1,2}^{2}\right)>0$, then the $q_{1,2}$ values w11l be Imaginary. In that event, only outgoing waves can be allowed as $z \rightarrow \pm \infty$. Such cases do not concern us in the present application.]

By definition, the linear $z$ velocity, $w^{-}$, and $z$ displacement, $z^{-}$, are related by

$1 \phi z^{\prime}=w^{\infty}$

Combining Equations ( 8 ) and (16), we obtain the expression

$$
\rho \phi^{2} z^{-}=D p^{-\star}=q P^{-\star} \text {, }
$$

a resuit that holds for both reglons 1 and 2 . Hence, at the interface of these respective reglons, we may write

$$
\rho_{1 \phi} \frac{2}{1} z_{11}=q_{1} p_{1}
$$

and

$$
P_{2} \phi_{2}^{2} z_{21}=q_{2} P_{21}
$$

where subscrlpt 1 denotes the interface. At the interface, the continulty conditions are 


$$
z_{11}=z_{21}
$$

and

$$
\text { Píl = Ṕ́三 . }
$$

Equations $(15 a, b),(18 a, b)$, and $(19 a, b)$ may be combined, along with the defintions of $\phi_{1,2}$, to yleld the desired dispersion relation

$$
\frac{\rho_{2} n^{2}}{\left[1-\frac{n^{2}}{k_{1}^{2} v_{2}^{2}}\right]^{1 / 2}}=\frac{-\rho_{1}\left(n+k_{1} u\right)^{2}}{\left[1-\frac{\left(n+k_{1} u\right)^{2}}{\left(k_{1}^{2} v_{1}^{2}\right)}\right]^{1 / 2}} .
$$

We nute in passing that the general dispersion relation for the hydrodynamic $v \in: s i o n$ of this problem reads

$$
\frac{\rho_{2^{n^{2}}}}{\left[1-\frac{\mathrm{n}^{2}}{\left(\mathrm{k}^{2} c_{2}^{2}\right)}\right]^{1 / 2}}=\frac{-\rho_{1}(\mathrm{n}+\mathrm{kU} \cos \theta)^{2}}{\left\lceil 1-\frac{(\mathrm{n}+k U \cos \theta)^{2}}{\left(\mathrm{k}^{2} c_{l}^{2}\right)}\right]^{1 / 2}},
$$

where $\theta$ is the angle between the wave vector $\underset{\sim}{k}=\underset{\sim}{1} k_{1}+\underset{\sim}{j} k_{2}$ and $\underset{\sim}{\mathrm{U}}$. Thus, to recover the general hydrodynamic resul $L$, we replace $v_{1,2}$ with $c_{1,2}, k_{1}$ with $k$, and $U$ with U $\cos \theta$ in Equation (20).

When we define the dimensionless quantities $x=n /\left(k_{1} v_{1}\right), a=\rho_{1} / \rho_{2}$, $b=\left(v_{1} / v_{2}\right)^{2}$, and Mach number $m=U / v_{l}$, Equation (20) becomes

$$
\frac{a(x+m)^{2}}{\left.\because-(x+m)^{2}\right]^{1 / 2}}=\frac{-x^{2}}{\left(1-b x^{2}\right)^{1 / 2}} \text {. }
$$


Equation (22) is of 6 th order in $x$. Af ter squaring both sides, and rearranging terms, we obtain

$$
\begin{aligned}
& \left(1-a^{2} b\right) x^{6}+2 m\left(1-2 a^{2} b\right) x^{5}+\left(m^{2}-6 a^{2} b m^{2}+a^{2}-1\right) x^{4} \\
& +4 m a^{2}\left(1-b m^{2}\right) x^{3}-m^{2} a^{2}\left(b m^{2}-6\right) x^{2}+4 a^{2} m^{3} x+a^{2} m^{4}=0
\end{aligned}
$$

Equation (23) has only one complex confugate pair of roots, which also satisfies Equation (22). These roots, which represent the $K-H$ modes, may be written as $x=x_{r} \pm 1 x_{1}$. The growing node has the negative sign, and its grouth rate is $\gamma=x_{1} k_{1} v_{1}=x_{1} k_{1}(U / m)$.

In the Incompressible Iimit $(\mathrm{m}=0)$, the roots of Equation (20) are

$$
n=-a k_{1} U \pm 1 \sqrt{\alpha(1-\alpha) k_{1} U}
$$

where $a=\rho_{1} /\left(\rho_{1}+\rho_{2}\right)$. The ratio of oscillatory frequency, $w$, to growth rate $\gamma$ is $\omega / \gamma=\left(\rho_{1} / \rho_{2}\right)^{1 / 2}$. The most rapid growth occurs when $\alpha=1 / 2$, corresponding to $a=1$. In this case, Equation (20) can be expanded easily in powers of $m$ to gauge the influence of compressibility on the growth rates. Thus, we obtain

$$
n \simeq-k_{1} \frac{U}{2} \pm 1 k_{1} \frac{U}{2}\left(1-\frac{m^{2}}{4}\right) .
$$

It is evident that compressibility reduces the unstable growth rates, which is to be expected on physical grounds. When $m=0.01$, the complex growth rate calculated from Equation (25) is identical to the corresponding numerical solution of Equation (23) to nine significant figures; when $m=0.20$, the results agree to four significant figures; when $m=1.00$, the growth rate predicted by Equation (25) is low by $\sim 7.5 \%$.

The growth rates of the unstable $K-H$ modes are shown in Fig. 3 for Gerwin's case; $a=b=1$, where we have defined $n \equiv \pm 1 \gamma+w$. These solutions can be derived analytically because Equation (23) reduces to a quintic, having one root $x_{5}=-m / 2$. The remaining quartic equation can be solved algebraically. As noted by Gerwin, ${ }^{3} \mathrm{~K}-\mathrm{H}$ instabilities can occur only when $m<\sqrt{8}$. As $\rho_{1} / \rho_{2}$ diminishes, the $K-H$ growth rates usualiy become smaller. 
Moreover, unless $v_{2} / v_{2}$ is very large, the range of $m$ values over which these instabilities can arise shrinks as well. This behavior is illustrated in Fig. 4. The curves represent models for which the undisturbed media are constrained by the condition $\rho_{1} v_{1}^{2}=\rho_{2} v_{2}^{2}$. If $v_{A}^{2} / c^{2} \gg 1, c^{2} / v_{A}^{2} \gg 1$, or $\gamma_{g}=2$, this condition amounts to requiring that regions 1 and 2 be in pressure equilibrium. [Strictly speaking, pressure equilibrium requires that $\left.\rho_{1}\left(c_{1}^{2} / \gamma_{g}+v_{A 1}^{2} / 2\right)=\rho_{2}\left(c_{2}^{2} / \gamma_{g}+v_{A}^{2} / 2\right).\right]$ Cases conforming to the unphysical condition, $\rho_{1} \mathrm{~V}_{1}=\rho_{2} \mathrm{v}_{2}$, are depicted in $\mathrm{Fig}$. 5. If this condition of "momentum equilibrium" is met, the growth rates remain relatively large as $\rho_{1} / \rho_{2}$ becomes small. In fhysical terms, the ratio of the "thermal" energy density of the fluid $i n$ region 1 to that in resion $2=v_{1} / v_{2}=\rho_{2} / \rho_{1}$. Consequently, ample energy is always available to drive the $K-\mathrm{H}$ instabilities. Figure 6 shows the normalized maximum rates (and corresponding Mach numbers) for "pressure equilibrium" models $\left(\rho_{1} v_{1}^{2}=\rho_{2} v_{2}^{2}\right)$ as a function of density contrast. Clearl, the growth rates of $K-H$ Instabilities diminish as the density contrast increases for reasonable physical models. Unlike the case for small $m$ Ifn which $\left.\omega / Y=\left(\rho_{1} / \rho_{2}\right)^{1 / 2}\right]$, the ratios of the corresponding oscillatory frequencies to these maximum growth rates are $\omega / \gamma_{\mathrm{m}} \sim 1$.

IV. INCOMPRESSIBLE MODELS WITH LINEAR SHEAR LAYERS

While the models of Section III have the virtue of including compressibility, they suffer from the shortcoming of imposing a velocity discontinuity between regions $i$ and 2 . Since shear viscosity was not included in these models, the shorter the Fourier wavelength, the more rapid will be the $\mathrm{K}-\mathrm{H}$ growth rate. However, if $\mathrm{K}-\mathrm{H}$ instabilities are responsible for the Kingfish striations, the observations show that a preferred wavelength is excited. As is the case when shear viscosity is present, the existence of a finite shear layer in a velocity profile will select a favored wavelength for which the $\mathrm{K}-\mathrm{H}$ grol:th rate is a maximum. From a physical viewpoint, shrar viscosity and velocity shear are related. If a discontinuous velocity profile is imposed upon a viscous fluid, then subsequently a shear layer will develop in the iluid through the action of shear viscosity. (Indeed, a linear velocity profile can be maintained in the presence of any constant coefficient of shear viscosity.)

For models having uniform, undisturbed densities, both $\mathrm{MP}^{2}$ and Chandrasekhar ${ }^{1}$ have shown that incompressible $K-H$ instabilities exporience a 


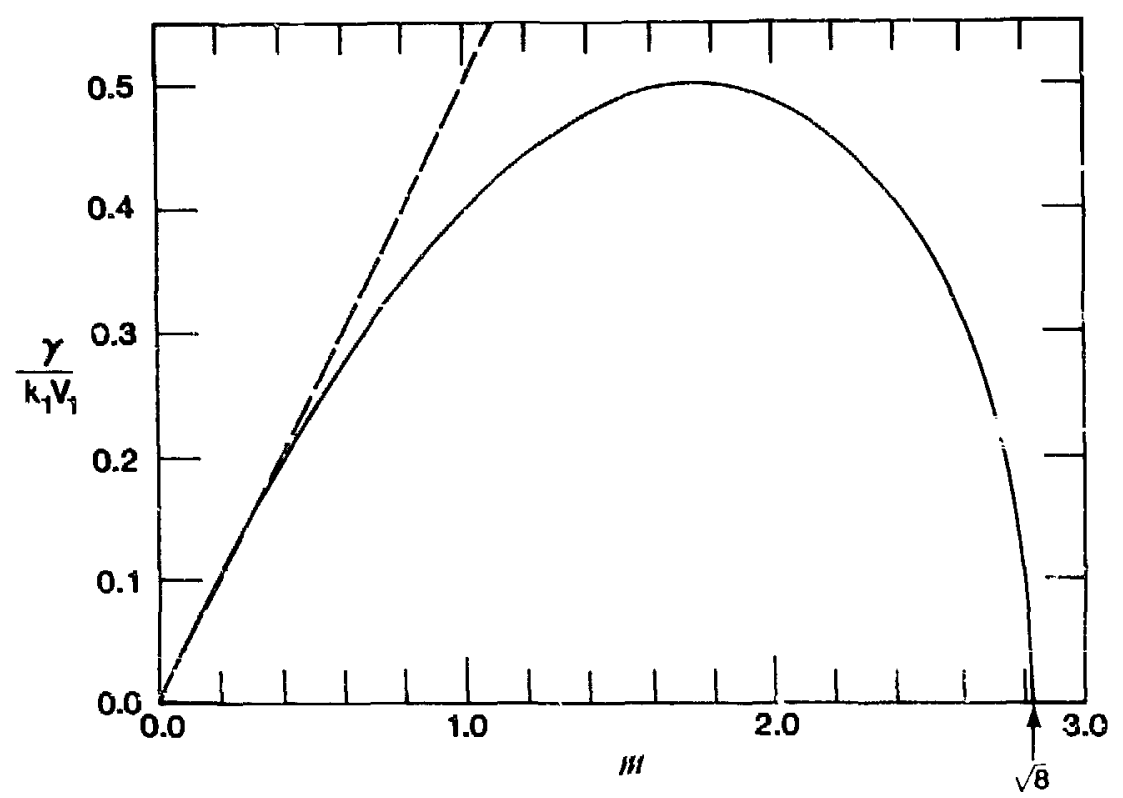

Fig. 3. Unstable growth rates in the Gerwin problem; $\rho_{1} / \rho_{2}=1$. The dashed line shows the incompressible growth rate, $y /\left(k_{1} v_{1}\right)=i n / 2$.

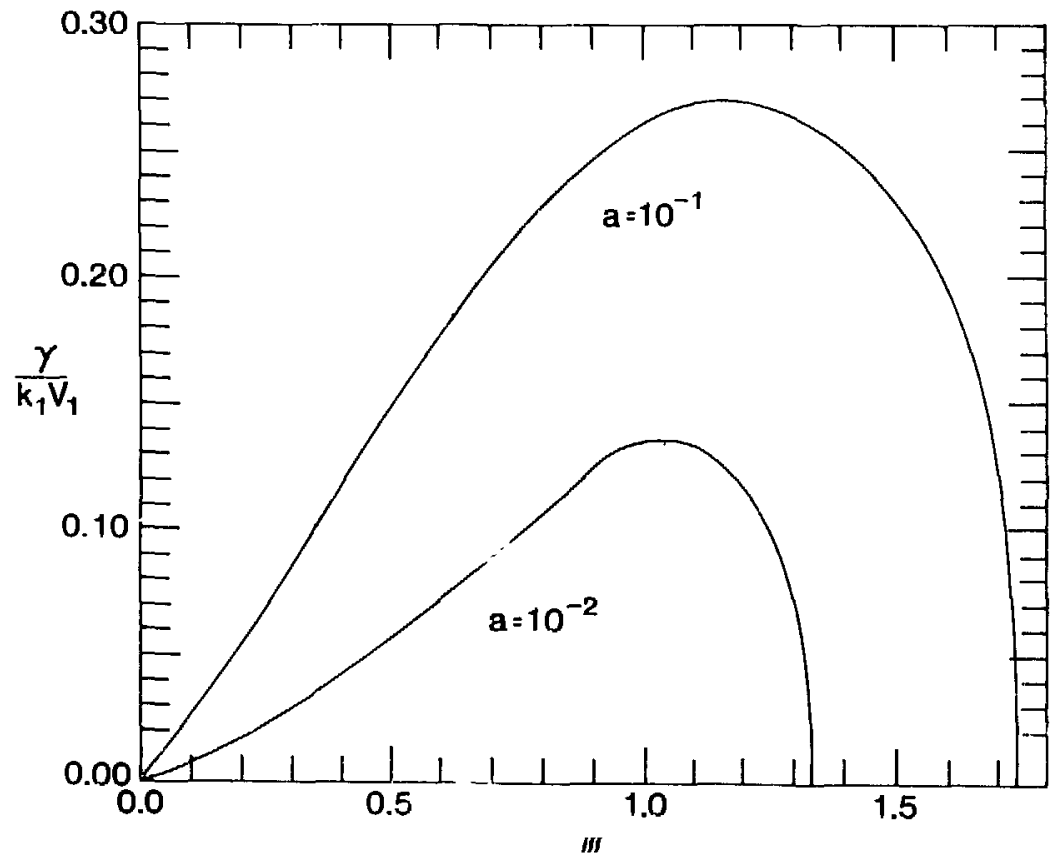

F1g. 4. Unstable growth rates in the generalized Gerwin problem when $\rho_{1} / \rho_{2}=10^{-1}, 10^{-2}$, and the media are $1 n$ "pressure equilibrium" $\left(\rho_{1} v_{1}^{2}=\rho_{2} v_{2}^{2}\right)$. 


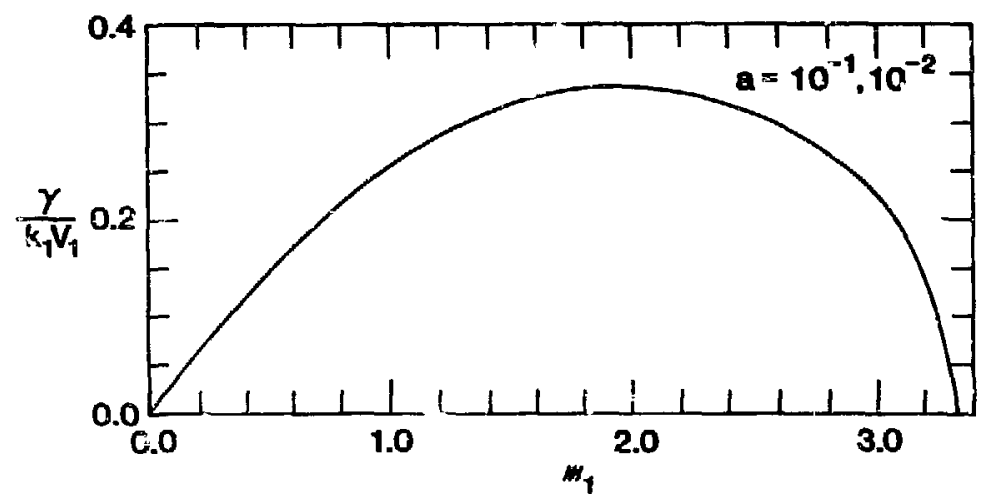

Fig. 5. Unstable growth rates in the generalized Gerwin problem when $\rho_{1} / \rho_{2}=$ $10^{-1}, 10^{-2}$, and $\rho_{1} v_{1}=\rho_{2} v_{2}$.

Fig. 6. Maximum unstable growth rates in the generalized Gerwin problem fin "pressure equilitirium" as a function of density contrast.

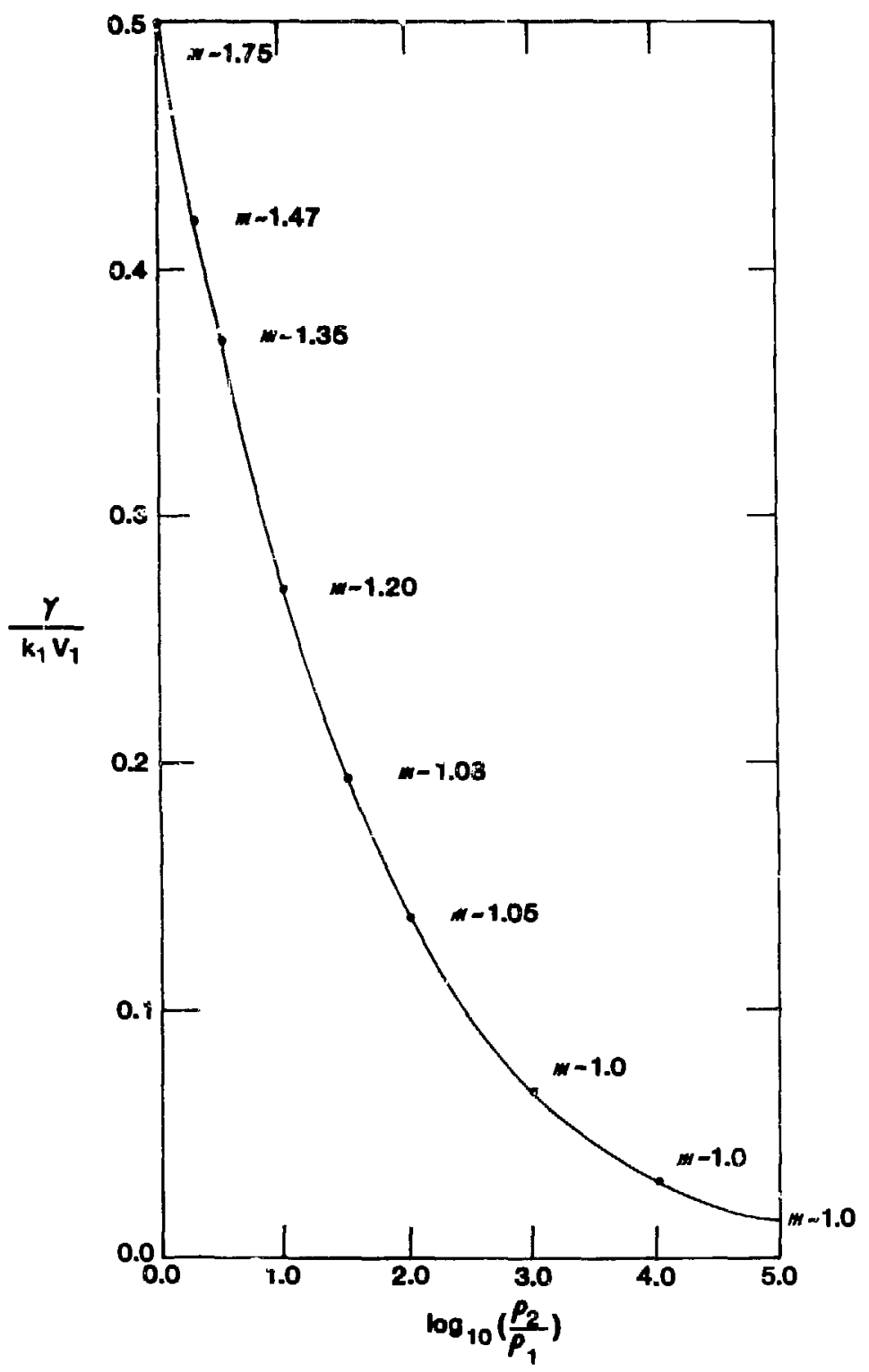


maximum growth rate when $k_{1} a \sim 1$, where a is the characteristic thickness of the shear layer. When compressibility is included, MP find that the optimal value of $k_{1} a$ decreases with increasing Mach number. In t.ieir models, which employed a hyperbolic tangent velocity profile, $K-H$ instabilities can occur only if $m<2$. (Recall that, for the discontinuous velocity profile, $m<\sqrt{8}$. ) As we would expect, the MP growth rates become smaller as $\mathrm{m}^{-1}$ diminishes (1.e., as compressibility becomes more important). Unfortunately, in the present context, the MP models do not allow for density stratification. However, a model that includes both velocity shear and density layering is illustrated in Fig. 7 .

We will consider only the incompressible case because (1) the growth rates are greatest in that limit and (2) the incompressible problem can be solved exactly and straightforwardly. Al though Chandrasekhar ${ }^{1}$ does not treat the density layering in enough detail for our application, he does provide an excellent outline of the solution to this problem ( $p .487$ ). For all three reglons in Fig. 7, the differential equation for $w^{-}$simplifies to

$$
\left(D^{2}-k_{l}^{2}\right) w^{\prime}=0
$$

Therefore, the solutions in the respective regions are

$$
w_{1}^{-}=A e^{-k} 1^{z}
$$

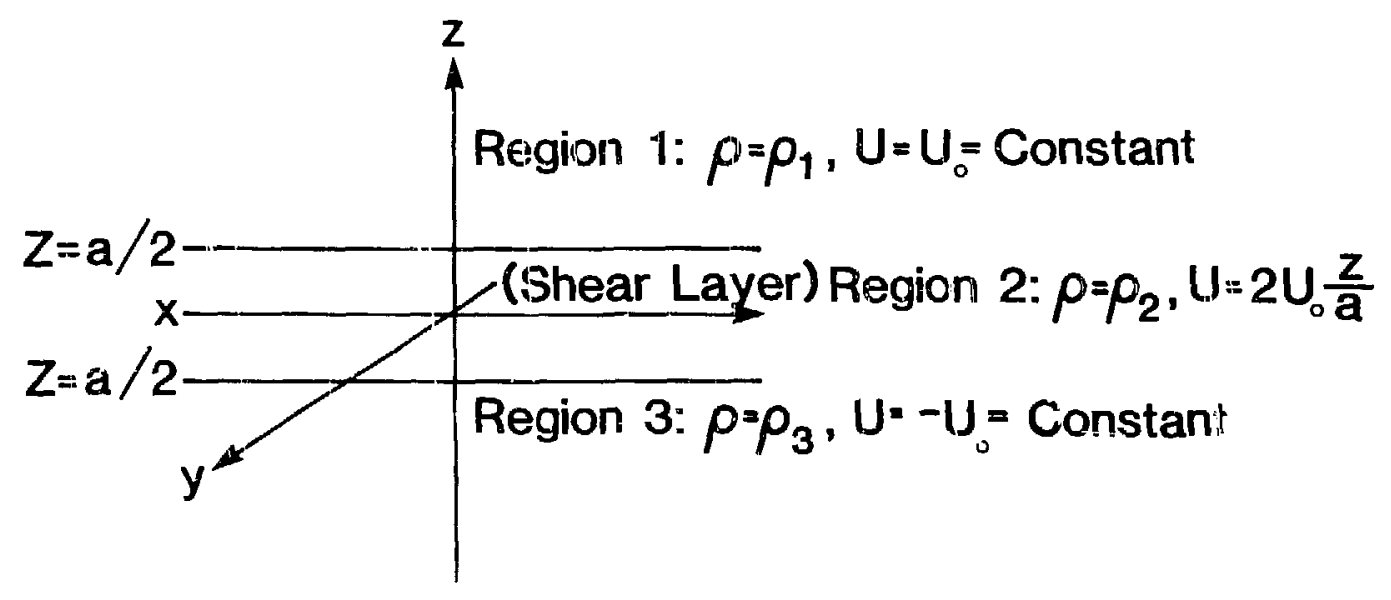

Fig. 7. The three-layer model with linear velocity shear. 


$$
w_{2}=A_{0} e^{-k_{1} z}+B_{0} e^{k_{1} z}
$$

and

$$
w_{3}^{-}=B e^{k_{1} z}
$$

where $A, A_{O}, B_{O}$, and $B$ are constants. At the Interface (1) between reglons 1 and 2 , the folluwing condition holds:

$$
\rho_{1}\left(n+k_{1} U_{0}\right) D w i-\rho_{2}\left(n+k_{1} U_{0}\right) D w_{21}+2 k_{1} \rho_{2}\left(\frac{U_{0}}{a}\right) w_{21}=0 \text {. }
$$

Moreover, the displacements must be continuous at the Interface;

$$
z_{11}^{\prime}=\frac{-1 w_{i 1}^{\prime}}{\left(n+k_{1} U_{0}\right)}=z_{21}^{\prime}=\frac{-1 w_{21}^{\prime}}{\left(n+k_{1} U_{0}\right)} \text {. }
$$

Consequently, at the interface,

$$
w_{11}^{r}=w_{21}^{r} .
$$

Using Eqs. (27a), (27b), (28), and (30), we obtain

$$
\frac{A_{0}}{B_{0}} e^{-k_{1} a}=-\frac{\left[\frac{2 U_{0}}{a}-\left(n+k_{1} U_{0}\right)-\frac{\rho_{1}}{\rho_{2}}\left(n+k_{1} U_{0}\right)\right]}{\left[\frac{2 U_{0}}{a}+\left(n+k_{1} U_{0}\right)-\frac{\rho_{1}}{\rho_{2}}\left(n+k_{1} U_{0}\right)\right]} .
$$

The Interface between reglons 2 and 3 may be treated similarly, yielding 


$$
\frac{B_{0}}{A_{0}} e^{-k_{1} a}=-\frac{\left[\frac{2 U_{0}}{a}+\left(n-k_{1} U_{0}\right)+\frac{\rho_{3}}{\rho_{2}}\left(n-k_{1} U_{0}\right)\right]}{\left[\frac{2 U_{0}}{a}-\left(n-k_{1} U_{0}\right)+\frac{\rho_{3}}{\rho_{2}}\left(n-k_{1} U_{0}\right)\right]}
$$

We define the following dinensionless quantities: $r_{1}=\rho_{1} / \rho_{2}, \quad r_{3}=\rho_{3} / \rho_{2}$, $x=k_{1}{ }^{a}$, and $y=n / k_{1} U_{0}$. In these variables, Equations (31) and (32) may be combined to form the quadratic equation

$$
\begin{aligned}
& {\left[\left(1+r_{1}\right)\left(1+r_{3}\right)-\left(1-r_{i}\right)\left(1-r_{3}\right) e^{-2 k}\right] y^{2}-\frac{2}{k}\left(r_{3}-r_{1}\right)\left(1-e^{-2 k}\right) y} \\
& -\left[\left(\frac{2}{k}-1-r_{1}\right)\left(\frac{2}{k}-1-r_{3}\right)-\left(\frac{2}{k}+1-r_{1}\right)\left(\frac{2}{k}+1-r_{3}\right) e^{-2 k}\right]=0 .
\end{aligned}
$$

For the uniform medium $\left(r_{1}=r_{2}=1\right)$,

$$
y= \pm k^{-1}\left[(k-1)^{2}-e^{-2 k}\right]^{1 / 2}
$$

a result originally due to Rayleigh (see reference 1). In this case, if Instabilities exist they contain no oscillatory component in the calculational frame. Instabilities exist if $\mathrm{e}^{-2 \kappa}>(k-1)$, implying that $k<\kappa_{c}=1.278465$. In order to demonstrate the connection between this three-layer problem and the incompressible, discontinuous case, we let $k \rightarrow 0$ in Equation (33), meaning that the shear layer is arbitrarlly thin in comparison with the perturbation wavelength. The resulting equation is

$$
y^{2}-2 \frac{\left(r_{3}-r_{1}\right)}{\left(r_{3}+r_{1}\right)}+1=0 .
$$

Letting $r_{1}=r_{3}^{-1}$, Equation (35) may be rewritten as

$$
y^{2}-2(1-2 \alpha)+1=0,
$$


where

$$
\alpha=\rho_{1} /\left(\rho_{1}+\rho_{3}\right) .
$$

Hence,

$$
y=(1-2 \alpha) \pm 2 i \alpha^{1 / 2} \sqrt{(1-\alpha)} .
$$

With respect to a rest frame in medium 3 (the fireball),

$$
\mathrm{n}=-a \mathrm{k}_{1} \mathrm{U} \pm 1 \sqrt{a(1-\alpha)} \mathrm{k}_{1} \mathrm{U}
$$

where $U=U_{1}-U_{3}=2 U_{0}$. This expression is identical with Eq. (24), the complex growth rate for an incompressible fluid with velocity discontinuity $U$.

The growth rates for the constant density case with linear velocity shear are shown in Fig. 8. The quantity $y_{1}(k)$ is the magnitude of the imaginary part of dimensionless angular frequency $y$. Also shown in Fig. 8 is the more conventional, normalized growth rate, ${ }^{2} \gamma a / U=y(k) k / 2$. Our solutions for this quantity are close to the MP solutions for the hyperbolic tangent velocity profile (shown in their Fig. 3). Graphs of ya/U for four different representative models are shown in Figs. 9-12. The dashed lines on these figuies show the incompressible, discontinuous growth rates. As was the case with our previous models, the growth rates diminish rapidly with increasing density contrast. Also, the wavenumber at which the growth is most rapid decreases slowly with increasing density contrast. Figure 13 depicts the maximum growth rate, as well as the corresponding values of $k$, as a function of total density contrast, $\rho_{3} / \rho_{1}$, for models having $\rho_{1} / \rho_{2}=\rho_{2} / \rho_{3}$. With respect to a rest frame in region 3 , the frequencies of oscillation are comparable to their corresponding maximum grosth rates. 

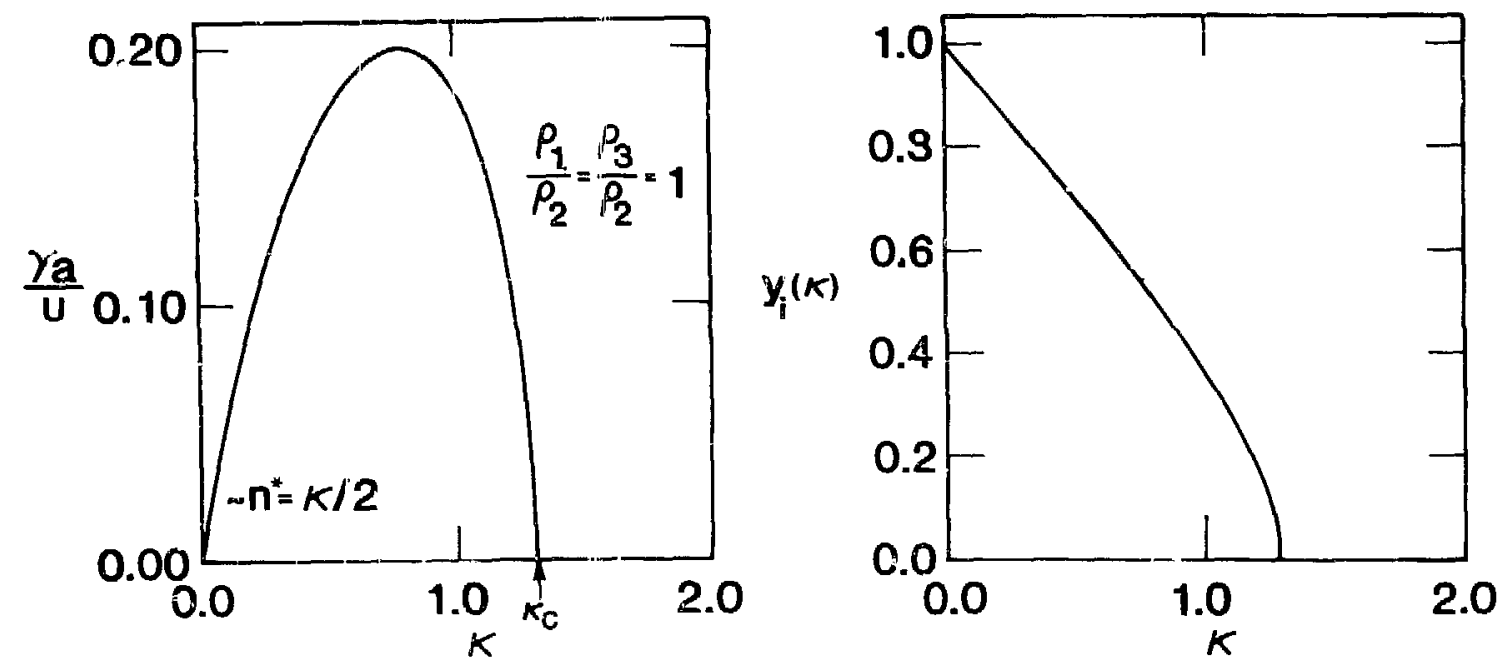

Fig. 3. Unstable growth râtes in the three-layer problem when $r_{1}=r_{3}=1$.

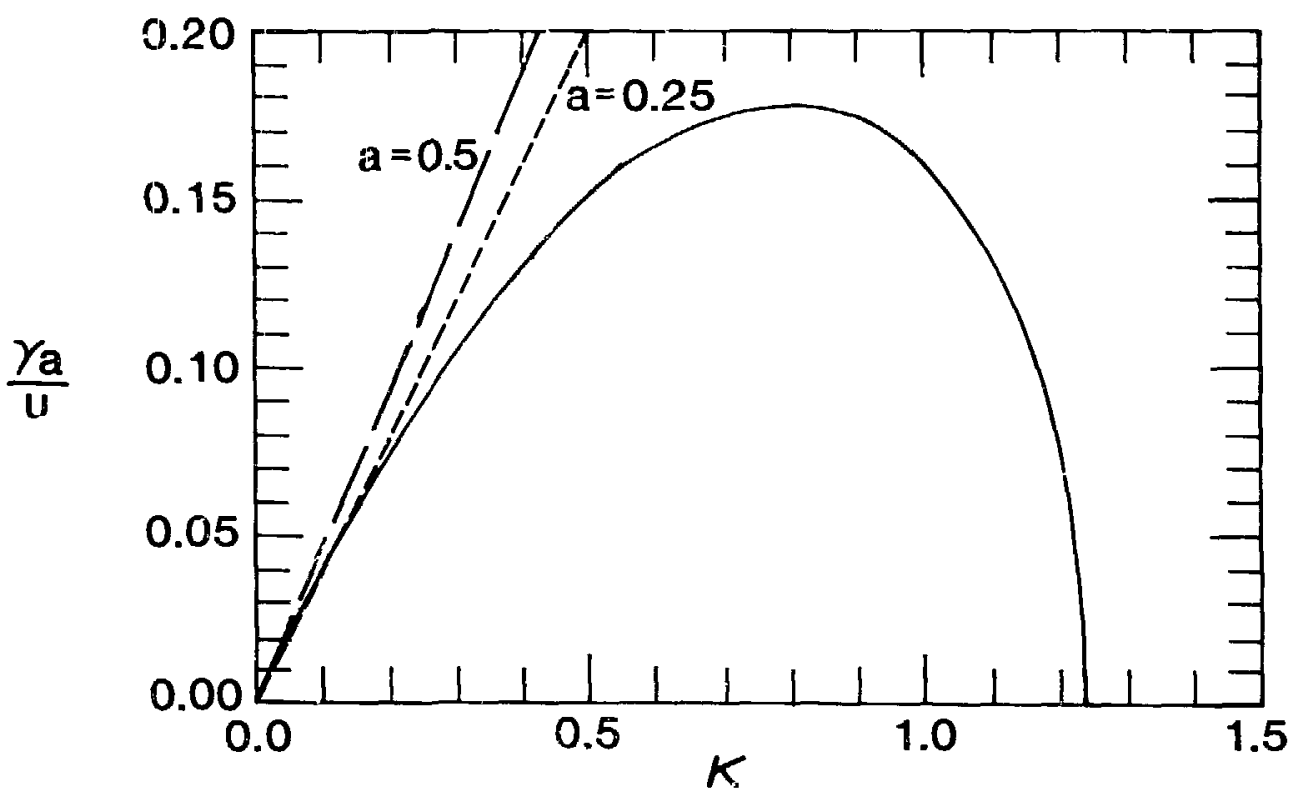

Fig. 9. Unistable growth rates in the three-layer problem when $r_{1}=1 / 2$ and $r_{3}$ $=2$. 


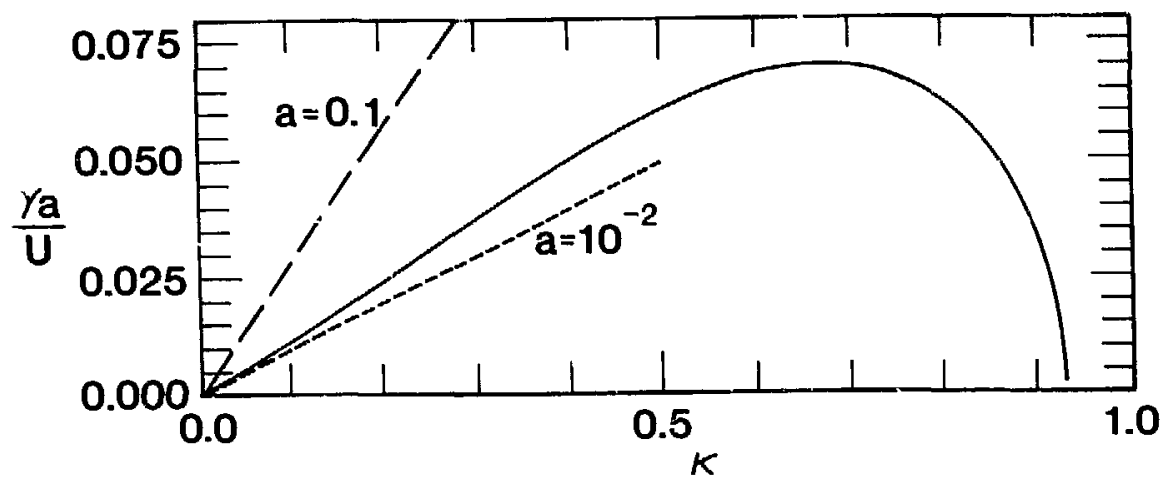

Fig. 10. Unstable growth rates in the three-layer problem when $r_{1}=10^{-1}$ and $r_{3}$ $=10$.

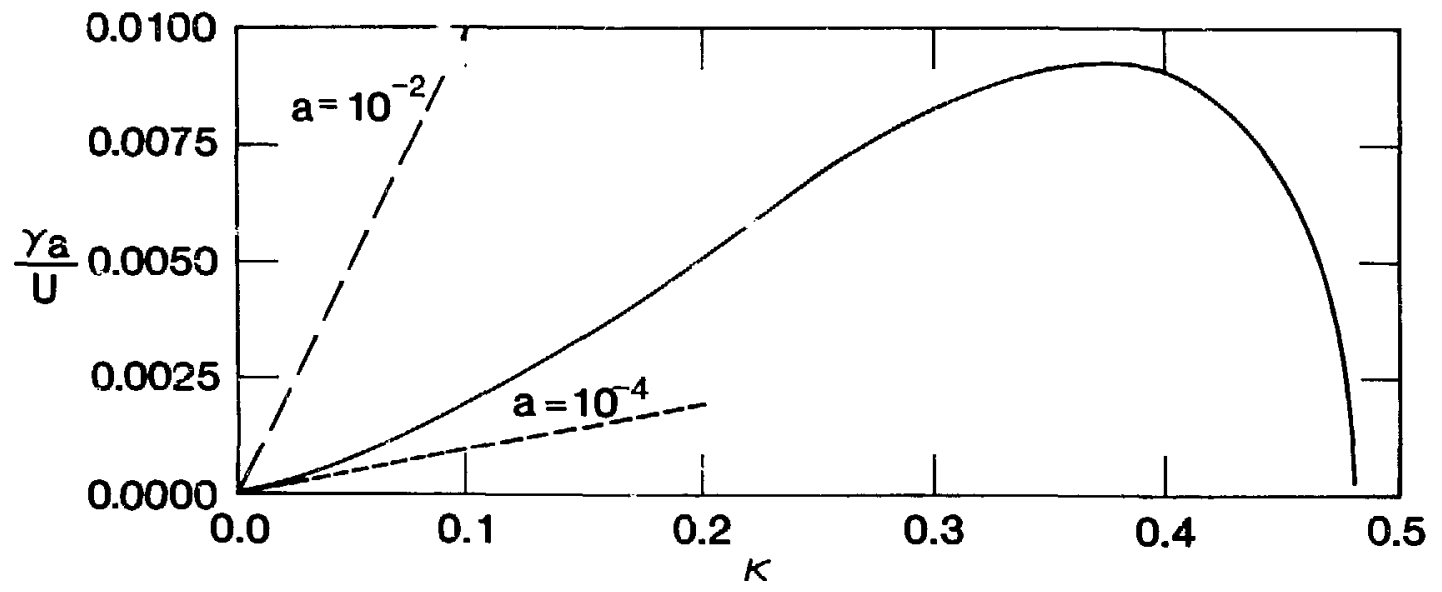

Fig. 11. Unstable growth rates in the three-layer problem when $r_{1}=10^{-2}$ and $r_{3}$ $=100$.

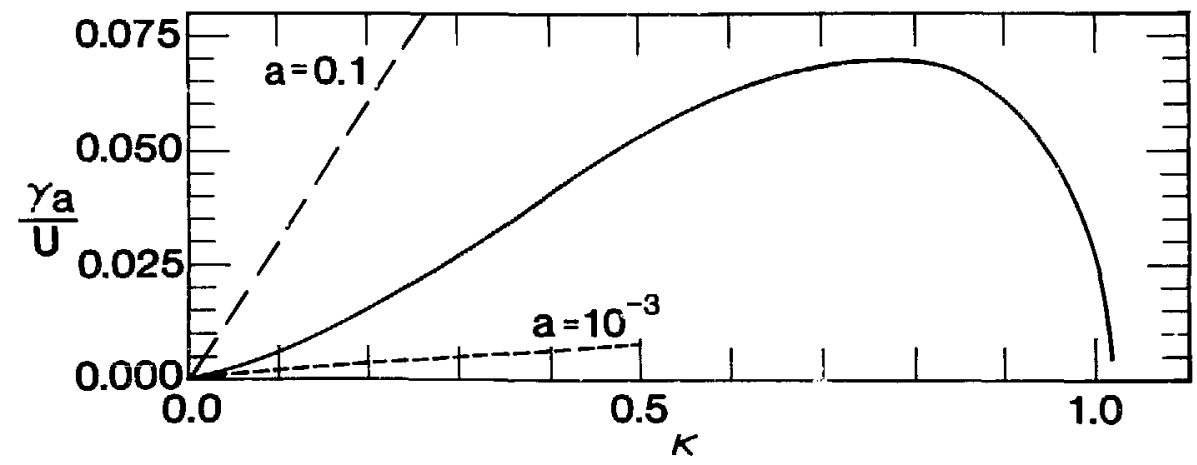

Fig. 12. Unstable growth rates in the three-layer problem when $r_{1}=10^{-3}$ and $r_{3}$ $=10$. 
Fig. 13. Maximum unstable growth rates in the three-layer problem as a function of $\rho_{3} / \rho_{1}$ when $\rho_{1} / \rho_{2}=$ $\rho_{2} / \rho_{3}$

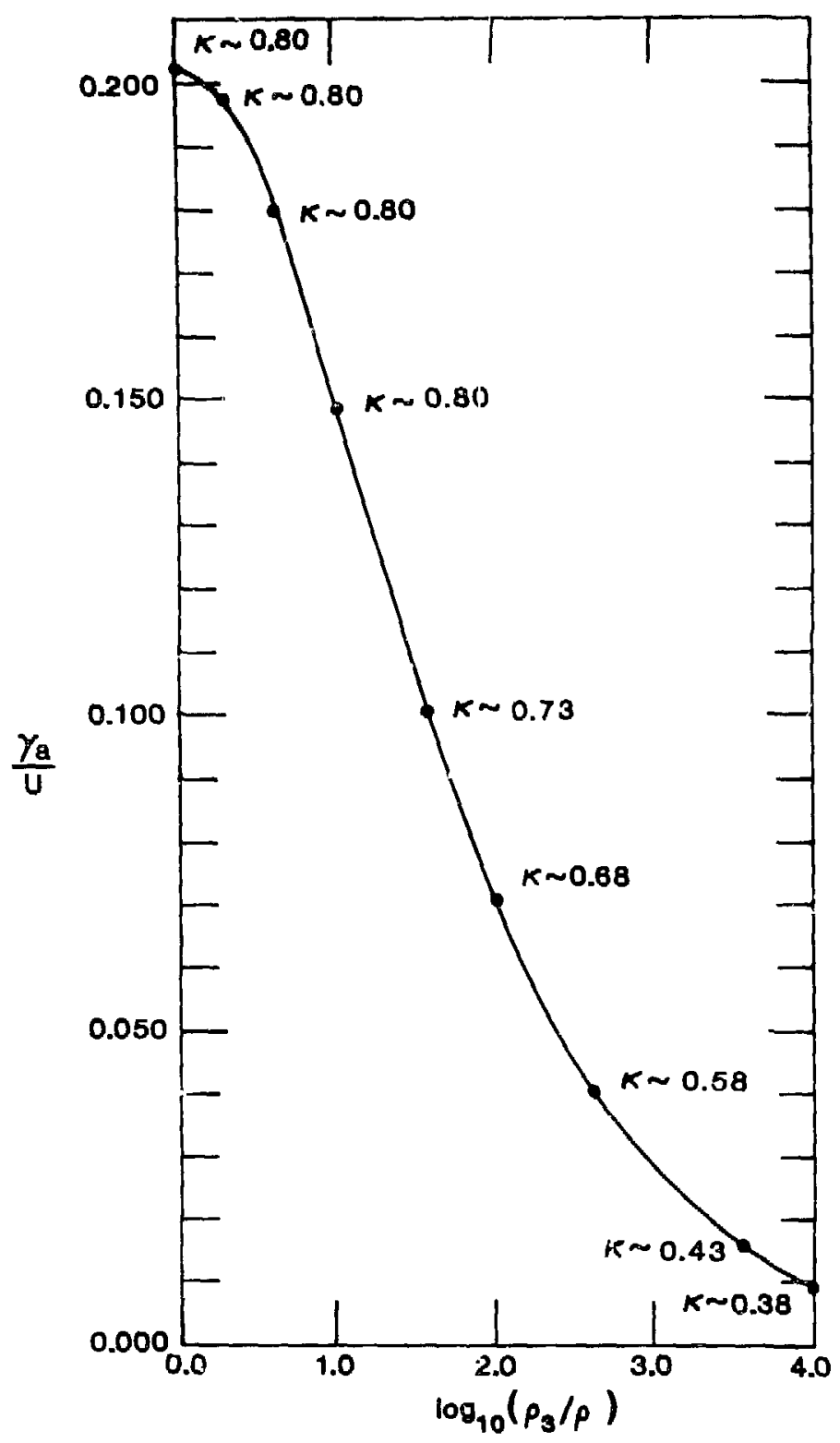

\section{REFERENCES}

1. S. Chandrasekhar, Hydrodynamic and Hydromagnetic Stab1l1ty, Sections 100, 101, 102, and 106 (Dover Publications, Inc., New York, 1981).

2. A. Miura and P. L. Pritchett, "Nonlocal Statility Analysis of the MHD Kelvin-Helmholtz Instability in a Compressible Plasma," J. Geophys. Res. 87, $7431(1982)$.

3. R. A. Gerwin, "Stability of the Interface Between Two Fluids in Relative Motion," Rev. Mod. Phys. 40,652 (1968). 